\title{
Investigating the Characteristics of Sit to Walk for Geriatric Residents of Assisted Living Facilities
}

\author{
Taylor Braden, $\mathrm{SPT}^{1}$, Madison Burtch, $\mathrm{SPT}^{1}$, Samantha Mott, $\mathrm{SPT}^{1}$, Alexandria Pyle, $\mathrm{SPT}^{1}$, \\ Lee Atkins, PT, MPT, PhD ${ }^{1}$, Heather Braden, PT, MPT, PhD, GCS ${ }^{* 1}$ \\ ${ }^{1}$ Dept. of Physical Therapy, Angelo State University, USA
}

\begin{abstract}
Purpose Assessing functional mobility is an important aspect of determining fall risk in the growing population of assisted living. Little is known about the sit-to-walk (STW) transition, especially what characteristics correlate with the duration it takes to perform a STW. Methods In this cross-sectional, exploratory study, 45 assisted living residents were evaluated for baseline measures: history of falls, assistive device (AD), lower-extremity strength, and balance (Tinetti POMA). The residents were then timed performing a STW, and, from these durations, were stratified into fast and slow groups. Statistical analysis was performed to determine relationships between the two duration groups and the baseline measures. Results Participants who had experienced a fall were significantly more likely to use an assistive device $(\mathrm{p}=$ 0.001). The use of an AD had a moderate negative correlation with composite strength $(\mathrm{r}=-0.428, \mathrm{p}=0.003)$. Duration of STW had a weak negative correlation with composite strength $(r=-0.299, p=0.046)$ and a moderate correlation with use of $\mathrm{AD}(\mathrm{r}=0.419, \mathrm{p}=0.004)$. Those with a faster duration of STW had significantly better balance $(\mathrm{p}=0.027)$. Conclusion These correlations support the need for healthcare professionals to address the use of adaptive equipment and physical training when evaluating functional mobility in assisted living residents. Providing appropriate equipment and improving overall strength in these individuals can help reduce their risk of falls. Individuals in assisted living facilities can be assessed similar to other geriatric populations, with special consideration on functional tasks and measures.
\end{abstract}

Key words Mobility, Transfers, Sit to walk, Sit to walk, Assisted living facility

Corresponding author Heather Braden (hbraden@angelo.edu)

Received date 17 Dec 2020

Revised date 02 Feb 2021

Accept date 14 Feb 2021

\section{Introduction}

Functional mobility is a daily challenge for the geriatric population in assisted living facilities (ALFs). Currently, one million Americans reside in ALFs. ${ }^{1)}$ Depending on the facility, they are not guaranteed access to mobility assistance at this level of care. The incidence of falls in the geriatric population is increasing since the geriatric population itself is increasing. It is estimated that by 2050, over one-fifth of the United States population will be over age 65. ${ }^{2}$ A 2014 study revealed that 28\% of Americans 65 and older reported a fall. ${ }^{3)}$ However, less than half of those who fall report the incident, so the real number is likely much

http:dx.doi.org/10.17817/2021.02.02.1111620 higher. ${ }^{3)}$ Sixty percent of emergency room visits and over half of injury-related deaths are the result of an older adult falling. ${ }^{4)}$ Falls continue to place a major financial burden on the healthcare system in the United States. ${ }^{3)}$ The annual cost of falls is estimated to be $\$ 50$ billion. ${ }^{5)}$ Aside from the physical and financial effects of falling, many people who experience a fall develop a subsequent fear of falling. This fear may cause them to limit their overall activity which can result in reduced strength and ambulatory mobility. When a person falls once, they are twice as likely to fall again. ${ }^{3)}$

Ambulatory mobility requires strength and balance to rise from a surface and walk to the target location before returning to a sitting position. Most falls tend to occur during everyday activities that challenge an individual's ambulatory mobility. ${ }^{6}$ More than slips and trips during ambulation, $41 \%$ of falls by older adults 
occur during transfers. ${ }^{7)}$ Various outcome measures predict fall risk by quantifying the different components of a transfer. This includes gait, static balance, and dynamic balance. Measures like the Tinetti Performance Oriented Mobility Assessment (POMA) and the Berg Balance Scale analyze a person's static and/or dynamic balance as a direct indication of potential future falls. ${ }^{89}$ ) The 30 second Sit to Stand Test and 5 Time Sit to Stand Test correlate fall risk with the ability and time it takes a person to simply rise from sitting without taking any additional steps. ${ }^{10,11)}$ Gait speed and Timed-Up-and-Go (TUG) tests correlate fall risk with the time a person takes to move a certain distance. $^{12,13)}$ Of these commonly administered balance assessments, only the TUG correlates the speed of the full transfer from sitting to walking with a risk for falls, but that transfer is not the only component of the TUG test. It also includes gait and the return to sitting in its analysis. ${ }^{13)}$ Less is available in the literature regarding fall risk during the segment of time in which a geriatric person begins motion from sitting to when they take an initial step forward (gait initiation). In this study, this segment of mobility is defined as sit to walk (STW).

Aspects of STW have been analyzed in other populations. One study analyzed the characteristics of this transition with and without a pause before gait initiation. ${ }^{14)}$ This study found that pausing before initiating gait requires less positional stability even in healthy individuals. Another study found that increases in angular velocity and linear accelerations during STW may predispose healthy, young individuals for balance loss. ${ }^{15)}$ Additionally, another study compared the characteristics of STW in healthy young and healthy elderly individuals. ${ }^{16)}$ They found differences in the center of pressure paths between the two groups of participants and concluded that the more varied center of pressure paths seen in the elderly participants were indicative of decreased postural stability. Analysis of community-dwelling older adults showed that a longer STW duration is associated with decreased lower extremity strength and poor balance control. ${ }^{17)}$

This study initially examined the time it takes for assisted living residents to perform a STW transfer. The researchers then stratified these participants into two groups, fast and slow risers, and looked for differences in characteristics of the two groups. Identifying strong relationships may help guide clinicians in fall prevention therapies and education. Since lower extremity muscle weakness and balance deficits have been widely shown to be risk factors for falls, it was hypothesized that decreases in muscle strength and balance would correlate to longer STW durations and assistive device (AD) use. ${ }^{18,19)}$

\section{Method}

\section{Subjects}

Participants were current residents of four ALFs in the Concho Valley. Each of these facilities gave permission to conduct research with the participants. The activities coordinators at the local ALFs were contacted in order to schedule a mobility screen on the community calendar so that participants could attend as able. The goal for this study was 50 participants. Volunteers for the research were not compensated. Inclusion criteria for this study was as follows: a resident of one of the ALFs, 60 years of age or older, and cognitively able to follow commands or instructions given by researchers. Exclusion criteria was provided to the activities coordinators prior to acquiring participants: unable to follow verbal commands or instructions, non-ambulatory, wheelchair or bed bound, lower extremity amputation without a prosthetic, and if the participant had any external lines or tubes. Participants could also be excluded at the researchers' discretion if deemed a safety or health risk, and such exclusions are reflected in the data collection. Table 1 demonstrates descriptive data of the participants in fast and slow groups.

\section{Procedure}

Two tests and measures, the Tinetti POMA and manual muscle testing (MMT), were performed during this study. These are both often used in the geriatric population to investigate balance, mobility, gait, and phys- 
Table 1. Descriptive Statistics for Participants from Each Group (Fast and Slow)

\begin{tabular}{|c|c|c|c|c|}
\hline & \multicolumn{2}{|c|}{ Fast (n=25) } & \multicolumn{2}{|c|}{ Slow $(n=20)$} \\
\hline & Mean (SD) & Range & Mean (SD) & Range \\
\hline Composite Strength (BW) & $0.76(0.26)$ & $(0.31,1.55)$ & $0.68(0.16)$ & $(0.34,1.02)$ \\
\hline Asymmetric Strength (BW) & $0.02(0.01)$ & $(0.01,0.04)$ & $0.02(0.01)$ & $(0.01,0.06)$ \\
\hline Tinetti Score & $23.52(2.87)$ & $(19.00,28.00)$ & $20.2(3.2)$ & $(15,26)$ \\
\hline Average Number Falls Per Person & $0.64(0.64)$ & $(0.00,2.00)$ & $1.11(1.85)$ & $(0.00,6.00)$ \\
\hline Age (Years) & $84.57(6.95)$ & $(71.00,94.00)$ & $88.22(5.08)$ & $(81.00,97.00)$ \\
\hline Gender Breakdown by Group & male $=5$ female $=20$ & $\mathrm{n} / \mathrm{a}$ & male $=3$ female $=17$ & $\mathrm{n} / \mathrm{a}$ \\
\hline $\begin{array}{c}\text { Number Subjects with History of } \\
\text { Falling }\end{array}$ & 14 & $\mathrm{n} / \mathrm{a}$ & 11 & $\mathrm{n} / \mathrm{a}$ \\
\hline Number of Subjects that Used AD & 17 & $\mathrm{n} / \mathrm{a}$ & 18 & $\mathrm{n} / \mathrm{a}$ \\
\hline
\end{tabular}

$\mathrm{SD}=$ standard deviation; range=(minimum, maximum); $\mathrm{BW}=$ body weights; $\mathrm{AD}=$ assistive device.

ical limitations. Research shows that using a handheld dynamometer to perform manual muscle testing of muscular strength has high reliability. ${ }^{20)} \mathrm{A}$ decline in muscle strength is highly related to an inability to maintain balance and increased risk of falls, especially the strength of knee flexion and extension. ${ }^{21,22)}$ The Tinetti POMA is commonly used to screen for risk of falling in the elderly population and has good interrater reliability and concurrent validity. ${ }^{8)}$

Before data collection began, participants were asked to read and sign a consent and demographic form which included: age, gender, leg length from popliteal fossa to heel, history of falls within the last year, and recent hospitalizations. Then, the participants were assigned a number and began the data collection process. Each of the researchers performed tests that they were specifically skilled in administering because they received previous training from a physical therapist. At each facility, the same researcher administered the same test to reduce error and bias in the data. MMT was performed on the participant's lower extremities to confirm strength and relate it to sit to walk trends. For the MMT, the participant was seated in a firm chair with armrests. Both lower extremities were tested in sitting using the standard procedure for the following: hip flexion, knee extension, knee flexion, ankle dorsiflexion, and ankle plantarflexion. Each MMT measurement in pounds was taken once and recorded.

Participants completed a Tinetti POMA, which gave the researchers an indication of their overall mobility and their fall risk. The Tinetti POMA test was administered by the same researcher at each assisted living facility to reduce error and bias in the data. The researcher explained the directions to the participant before administering the test. When testing the balance section of the test, the patient was seated in the same firm chair used during the MMT to begin. For the gait section of the test, the patient stood with the researcher and walked across the room (independently or with their usual assistive device), first at a usual pace, and then at a rapid but safe pace. ${ }^{23)}$ The researcher provided verbal instructions and observed the performance. Scores were totaled and recorded. Based on the scores and fall risk categories, the researchers were able to indicate the risk of falls for each participant.

The testing chair was adapted in height with dense foam so that all participants sat at a knee angle of $120 \%$ of the participant's lower leg length (popliteal fossa to the floor). The participant stood up with no back support to rise from the chair and, if needed, their $\mathrm{AD}$ was placed within reach to use for the activity. They were instructed to walk to a cone 10 feet away, following the verbal command "go" given by the researcher. The task was designed to simulate 
meaningful reasons for rising from a chair to walk, such as answering a phone or doorbell or rising to exit in the event of an emergency. Data was collected from the Tekscan pressure mat that was placed under the participant's feet. The dependent variable, time in seconds, for each participant was measured by the Tekscan software. Time was measured from when the researcher gave the verbal command to go until the moment the participant's feet left the mat. The time was recorded and saved on a secure hard drive. Composite strength scores were calculated for each participant and divided by their body weight $(\mathrm{kg})$. Composite strength is the average of left and right of all manual muscle tests summed together. A score of strength asymmetry was assessed by averaging the relative difference of all left and right strength measures. Participants were stratified into fast and slow duration groups based upon completion time of the STW activity (rise from chair and the first step for the walking task) in which the participant was instructed to stand up and go touch the target.

\section{Statistical Analysis}

In SPSS (Version 25), the researchers utilized a Shapiro-Wilk test for normality. To determine a difference between means, a Mann-Whitney $U$ test was completed. The researchers stratified participants into fast versus slow sit to walk (STW) groups and compared means to look for significant differences in their Tinetti POMA score, strength score relative to participant weight (both composite and asymmetry difference of left versus right leg), gender, age, use of $\mathrm{AD}$, and history of falls. After discovering the significant differences in the fast versus slow STW groups, the researchers were interested in any associations the variables had to one another or strength and direction of relationship between variables. Researchers computed a composite strength score for each participant and a score of strength asymmetry. Pearson point-biserial correlations were run for one nominal and one ratio variable such as duration and $\mathrm{AD}$ and quantity of falls and $\mathrm{AD}$. Spearman rank tests were run for associations between two ratio variables such as duration and composite strength, or ordinal data, such as the Tinetti Performance Oriented Mobility Assessment (POMA) score, and one other variable. The McNemar test was completed for two nominal variables such as falls and AD. Those with significance $(p<.05)$ have a strength and direction of monotonic relationship of the given correlation coefficient for the 2 variables considered as follows: .1-.3 weak correlation, >.3-.7 moderate correlation, and >.7-.9 strong correlation. ${ }^{24)}$

\section{Results}

A natural division occurred between those in the fast STW group ( $\leq 4.31$ seconds) and the slow STW group ( $\geq 5.14$ seconds). The mean of the fast group $(n=25)$ that completed the STW task was 2.83 seconds (.76 $\mathrm{SD})$. The mean of the slow group $(\mathrm{n}=20)$ that completed the STW task was 8.74 seconds (2.43 SD). Because the Shapiro-Wilk tests for normality produced $\mathrm{p}<.05$, the data lacked normal distribution. Thus, non-parametric statistics were utilized with significance lev$\mathrm{el}$ at $\mathrm{p}<.05$. Using a Mann-Whitney $\mathrm{U}$ test (nonparametric, independent samples) (Table 2), a significant difference in the means occurred for duration (fast versus slow group) and the Tinetti POMA score $(p=.018)$ such that the fast group had significantly better Tinetti POMA scores than the slow duration group. Results also showed a significant difference in duration and use of an $\mathrm{AD}(\mathrm{p}=.027)$ such that those in the fast STW group were less likely to use an AD. Three different correlations were completed to observe associations between variables. Pearson point-biserial correlations were completed (one nominal and one ratio variable, $\mathrm{p}<.05)$, such as duration and $\mathrm{AD}(\mathrm{r}=.419$, $\mathrm{p}=.004)$, so faster STW and no AD were moderately correlated. Likewise, composite strength and use of

\section{Table 2: Test Statistics}

$\begin{array}{cccc}\text { Variable } & \text { Mann-Whitney U } & Z & \begin{array}{c}\text { Significance } \\ \text { (2-tailed) }\end{array} \\ \text { Tinetti } & 149.500 & -2.364 & .018^{*} \\ \begin{array}{c}\text { Assistive } \\ \text { Device }\end{array} & 183.000 & -2.207 & .027^{*} \\ \text { *Statistically significant (Asymp. Sig., 2-tailed) } & \end{array}$


AD were moderately negatively correlated ( $r=-.428$, $\mathrm{p}=.003$ ), demonstrating that those with AD have weaker strength. Spearman rank tests were completed to show associations between two ratio variables, so duration and composite strength had a weak negative correlation $(r=-.299, p=.046)$. The McNemar test was completed for two nominal variables. The McNemar test demonstrated that those who fall are also significantly more likely to use an $\mathrm{AD}(\mathrm{p}=.001)$.

\section{Discussion}

As the geriatric population continues to rise, more people are transitioning to ALFs in order to improve their quality of life. ALFs have grown in popularity across the country, but there is little research regarding mobility and safety in ALFs compared to other populations. This highlights the need for further research to address the mobility, exercise, function, general health, and quality of life of those in ALFs. The purpose of this study was to fill the gaps where research is needed for the geriatric population in ALFs. The researchers hypothesized that participants with a lower Tinetti Balance score would have a higher total STW time. Our analysis supported this hypothesis: individuals with a lower Tinetti score took longer to complete the STW task, as seen in Table 3. A lower Tinetti score reflects increased fall risk. In a study by Cheng et al, researchers studied a similar task. The authors timed patients with stroke and healthy patients performing a sit to stand and found that pa- tients with stroke who had fallen had increased time to perform a sit to stand transfer compared to patients with stroke who had not fallen and healthy controls. ${ }^{25)}$

Each of these correlation results provide both strength and direction of a monotonic relationship of correlation coefficients for two compared variables considered. Some other variables assessed during data collection included fall risk, use of an $\mathrm{AD}$, duration of STW transition, and composite strength. Our analysis found significant correlations between $\mathrm{AD}$ use and slower STW time, as well as AD use and decreased overall composite strength. Clinical application of these results can help the geriatric population with their functional mobility, fall risk, and general well-being in regard to movement and safety. Fall risk is an important factor to analyze in this population. Our results indicated there was a significant relationship between the participants with a history of falls and those who used ADs for ambulation. Research from Pellicer et al (2020) supports this finding, reporting that use of ADs was highly correlated with falls, and that these falls occur when the individuals are not using them. ${ }^{26)}$ This emphasizes the importance of education for ALF residents, which was a goal of the study. When the Tinetti test was completed and the individuals were deemed high, moderate, or low fall risk, they were educated on what that meant and were given five tips for preventing falls in their rooms.

The slower transitioners who had weaker overall composite strength were moderately related to use of

Table 3: Spearman Rank Correlation for Ordinal Data

\begin{tabular}{|c|c|c|c|c|c|c|c|}
\hline & Composite Strength & Duration & Tinetti & Assistive Device & Gender & Age & Fast Duration Split \\
\hline Composite Strength & 1.000 & $-0.299^{*}$ & 0.092 & $-0.325^{*}$ & -0.139 & 0.141 & -0.260 \\
\hline Duration & $-0.299^{*}$ & 1.000 & $-0.514^{*}$ & $0.519^{*}$ & 0.022 & 0.208 & $0.866^{*}$ \\
\hline Tinetti & 0.092 & $-0.514^{*}$ & 1.000 & -0.292 & -0.070 & -0.104 & $-0.356^{*}$ \\
\hline Assistive Device & $-0.325^{*}$ & $0.519^{*}$ & -0.292 & 1.000 & $0.311^{*}$ & -0.041 & $0.333^{*}$ \\
\hline Gender & -0.139 & 0.022 & -0.070 & $0.311^{*}$ & 1.000 & $-0.352^{*}$ & -0.106 \\
\hline Age & 0.141 & 0.208 & -0.104 & -0.041 & $-0.352^{*}$ & 1.000 & 0.226 \\
\hline Fast Duration Split & -0.260 & $0.866^{*}$ & $-0.356^{*}$ & $0.333^{*}$ & -0.106 & 0.226 & 1.000 \\
\hline
\end{tabular}

$\mathrm{N}=45$ (exception: for age, $\mathrm{N}=41$ )

${ }^{*}$ Correlation is significant at the 0.05 level (2-tailed) 
an AD. This can be applied clinically as well. Physical function in this population is important for independence, safety, and functional mobility. One study discussed how aerobic exercise, muscle-strengthening, and multicomponent physical activity have the strongest relationship to improvements in physical function in the general aging population and in those with chronic conditions. ${ }^{27}$ This study found that weaker participants were more likely to use an $\mathrm{AD}$, walk slowly, and experience falls. Therefore, it can be clinically relevant to work on strengthening of core and lower body ${ }^{28)}$ and gait speed to help with the prevention of falls. ${ }^{27)}$ Another study found that physical activity can help reduce serious fall-related injuries by $32-40 \% .{ }^{29)}$ Visual feedback video interventions also improve balance during movements in the elderly. ${ }^{30)}$ As the population ages and becomes an increased risk for fall-related injuries, physical activity can be an effective tool in delaying and reducing the loss of muscle strength and physical function.

This study has limitations that need to be acknowledged. The study received 45 participants from four facilities in San Angelo, Texas. The standardized chair had armrests that did not come to the front of the chair and some participants had to reach back further than desired to use the arm rests to push off during the STW transfer. Therefore, the findings of the current study may not be generalizable when using chairs without armrests or chairs with armrests in a different position. Due to the size of the Tekscan mat under the participants' feet, more than one attempt was occasionally required to step fully off of the mat. This may have influenced the results of the study as it caused some participants to take larger steps than they would in their natural STW pattern.

As previously mentioned, there is currently little research on geriatric residents in ALFs. This study was able to expand the literature and provide a starting point for other research. Future studies should examine the relations between sit to walk strategies including hand use and their influence on gait and balance during transfers and walking. Future studies could also examine the relationships between STW strategy and sway, ground reaction force, or center of pressure.

Currently, about one million Americans reside in ALFs, and this number is only expected to rise as the geriatric population continues to grow. Older individuals are at increased risk of fall-related injuries. This study showed that duration of STW and strength are moderately correlated to use of an assistive device. Specifically, duration of STW is negatively associated with composite strength, and those who have experienced falls are more likely to use an assistive device. These findings allow health care professionals to consider the need for adaptive equipment and physical training for older adults with strength deficits. With improved overall strength and use of an $\mathrm{AD}$, older individuals in may improve their physical functioning.

\section{Funding resource}

This research was funded by an internal enhancement grant at Angelo State University (\#0010-45161). Grant entities had no role in the research design, data collection, or manuscript.

\section{References}

1. American Senior Communities. Assisted Living Statistics and Facts. 19 August 2019. Accessed 27 June 27, 2020. https://www.asccare.com/assisted-living-statistics-a-dee per-dive-into-the-demographics/.

2. National Council on Aging. Falls Prevention Facts. 4 June 2018. Accessed 2 April 2019.

https://www.ncoa.org/news/resources-for-reporters/get-t he-facts/falls-prevention-facts/.

3. CDC Injury Center. Home and Recreational Safety 11 October 2016. Accessed 2 April 2019.

https://www.cdc.gov/homeandrecreationalsafety/falls/in dex.html.

4. Haddad YK, Bergen G, Luo, F. Reducing fall risk in older adults: Evidence supports addressing medication management. Am J Nurs. 2018;118(7):21-2.

5. Florence CS, Bergen G, Atherly A, et al. Medical costs of fatal and nonfatal falls in older adults. J Am Geriatr Soc. 2018;66(4):693-8.

6. Aberg AC, Frykberg GE, Halvorsen K. Medio-lateral stability of sit-to-walk performance in older individuals 
with and without fear of falling. Gait Posture. 2010;31(4):438-43.

7. Robinovitch SN, Feldman F, Yang Y, et al. Video capture of the circumstances of falls in elderly people residing in long-term care: an observational study. The Lancet. 2013;381(9860):47-548.

8. Raîche M, Hébert R, Prince F, et al. Screening older adults at risk of falling with the Tinetti balance scale. The Lance. 2000;356(9234),1001-2.

9. Park SH, Lee YS. The diagnostic accuracy of the Berg Balance Scale in predicting falls. West J Nurs Res. 2017;39(11):1502-25.

10. Zhang F, Ferrucci L, Culham E, et al. Performance on five times sit-to-stand task as a predictor of subsequent falls and disability in older persons. J Aging Health. 2013;25(3):478-92.

11. McAllister LS, Palombaro KM. Modified 30-second sit-to-stand test: Reliability and validity in older adults unable to complete traditional sit-to-stand testing. J Geriatr Phys Ther. 2020;43(3):153-8.

12. Middleton A, Fritz SL, Lusardi M. Walking speed: the functional vital sign. J Aging Phys Act. 2015;23(2): 314-22.

13. Barry E, Galvin R, Keogh C, et al. Is the timed up and go test a useful predictor of risk of falls in community dwelling older adults: a systematic review and meta-analysis. BMC Geriatr. 2014;14:1-14.

14. Jones GD, James DC, Thacker M, et al. Parameters that remain consistent independent of pausing before gait-initiation during normal rise-to-walk behaviour delineated by sit-to-walk and sit-to-stand-and-walk. PLoS One. 2018;13(10):1-14.

15. Kondilopoulos N, Rousanoglou EN, Boudolos KD. Inertial sensing of the motion speed effect on the sit-to-walk activity. Gait Posture. 2018;61:111-6.

16. Bestaven E, Petit J, Robert B, et al. Center of pressure path during sit-to-walk tasks in young and elderly humans. Ann Phys Rehabil Med. 2013;56(9-10):644-51.

17. Chen T, Chou L. Effects of muscle strength and balance control on sit-to-walk and turn durations in the timed up and go test. Arch Phys Med Rehabil. 2017;98(12): 2471-6.

18. Moreland JD, Richardson JA, Goldsmith $\mathrm{CH}$, et al. Muscle weakness and falls in older adults: A systematic review and meta-analysis. J Am Geriatr Soc. 2004;52(7):1121-9.
19. Wang D, Zhang J, Sun Y, et al. Evaluating the fall risk among elderly population by choice step reaction test. Clin Interv Aging. 2016;11:1075-82.

20. Suzuki, T. Reliability of measurements of knee extensor muscle strength using a pull-type hand-held dynamometer. J. Phys. Ther. Sci. 2015;27(3):967-71.

21. Pizzigalli L, Filippini A, Ahmaidi S, et al. Prevention of falling risk in elderly people: The relevance of muscular strength and symmetry of lower limbs in postural stability. J. Strength and Cond. Res. 2011;25(2), 567-74.

22. Ding, L, Yang, F. Muscle weakness is related to slipinitiated falls among community-dwelling older adults. J Biomech. 2016;49(2), 238-43.

23. Tinetti ME, Williams TF, Mayewski R. Fall Risk Index for elderly patients based on number of chronic disabilities. Am J Med. 1986;80:429-34

24. Akoglu H. User's guide to correlation coefficients. Turk. J. Emerg. Med. 2018;18(3):91-3.

25. PT, MY L, MK W, et al. The sit-to-stand movement in stroke patients and its correlation with falling. Arch. Phys. M. 1998;79(9),1043-46.

26. Pellicer-García B, Antón-Solanas I, Ramón-Arbués E, et al. Risk of falling and associated factors in older adults with a previous history of falls. Int $\mathrm{J}$ Environ Res Public Health. 2020;17(11):E4085.

27. Puts MT, Toubasi S, Andrew MK, et al. Interventions to prevent or reduce the level of frailty in community-dwelling older adults: A scoping review of the literature and international policies. Age Ageing. 2017;46(3):383-392.

28. Mun DJ, Lee SH. Effect of body stabilization on trunk muscle activity in adults. J Korean Soc Neurother. 2019:23(3):1-7.

29. Dipietro L, Campbell WW, Buchner DM, et al. Physical activity, injurious falls, and physical function in aging: An umbrella review. Med Sci Sports Exerc. 2019;51(6): 1303-13.

30. Lee, SH, Kim, YH, Park, SK, et al. The effect of visual feedback training using Wii Fit on balance for the elderly. J Korean Soc Neurotherapy. 2013:17(1):7-14. 
